\title{
Cultivares brasileiras de soja: características para a produção de tofu e aceitação pelo mercado consumidor
}

\author{
Brazilian soybean cultivars: tofu-making characteristics and \\ acceptance by consuming market
}

\author{
Vera de Toledo Benassi ${ }^{1 *}$; Marta de Toledo Benassi²; Sandra Helena Prudencio ${ }^{2}$
}

\begin{abstract}
Resumo
Neste trabalho, oito cultivares de soja brasileira foram avaliadas quanto ao seu potencial para produção de tofu e os produtos finais do processamento foram submetidos à aceitação sensorial. Os grãos das cultivares apresentaram diferenças em termos de tamanho (11-22 g/100 grãos), conteúdo de cascas (6,3-8,3 g casca/100 g grãos) e capacidade de absorção de água (121-140 g água/100 g grãos). Todas as cultivares foram semelhantes no rendimento em tofu (média de $271 \mathrm{~g}$ tofu/100g grãos), mas os produtos resultantes apresentaram dureza distinta (2,3-3,8 N). As médias obtidas no teste de aceitação de atributos evidenciaram diferenças entre os tofus, em termos de sabor (notas entre 5,3 e 6,2) e textura (notas entre 5,6 e 6,4), mas não na aceitação global (média de 5,8). O Mapa de Preferência Interno mostrou uma segmentação dos consumidores em três grupos distintos. Esta informação pode nortear uma empresa fabricante de tofu na escolha da matéria-prima, definindo uma ou mais cultivares de soja que melhor atendam às expectativas dos consumidores.

Palavras-chave: Tamanho do grão, conteúdo de cascas, rendimento de tofu, textura, preferência, segmentação de consumidor
\end{abstract}

\begin{abstract}
In this investigation, eight Brazilian soybean cultivars were evaluated with respect to their tofu-making potential and final products underwent sensory acceptance. Grains from each cultivar showed differences related to size, seedcoat content and water absorption capacity. All cultivars displayed similar tofu yield, but resultant products presented distinct hardness. Mean values obtained on attribute acceptance testing showed tofu were different in terms of flavor and texture but not in global evaluation. Internal Preference Mapping indicated consumer segmentation in three different groups. This information could guide tofu manufacturers in raw material selection, in order to define one or more soybean cultivars that better satisfy the expectations of the consumers.

Key words: Soybean size, soybean seedcoat content, tofu yield, tofu texture, MDPREF, consumer segmentation
\end{abstract}

\footnotetext{
${ }^{1}$ Engenheira de Alimentos e Mestre em Tecnologia de Alimentos pela UNICAMP. Doutoranda no Dept ${ }^{0}$ de Ciência e Tecnologia de Alimentos da Universidade Estadual de Londrina, UEL. Pesquisadora da Embrapa. E-mail: vera.benassi@gmail.com

${ }^{2} \operatorname{Prof}^{\mathrm{a}} \mathrm{Dr}^{\mathrm{a}}$ do Dept ${ }^{\mathrm{o}}$ de Ciência e Tecnologia de Alimentos da Universidade Estadual de Londrina, UEL. E-mail: martatb@uel.br; sandrah@uel.br

* Autor para correspondência
} 


\section{Introdução}

Embora a produção de tofu não seja uma tecnologia nova, produzir tofu com excelente qualidade tecnológica e sensorial continua sendo um desafio. Para os fabricantes de tofu, o rendimento é importante do ponto de vista econômico, assim como a qualidade do produto, na qual a textura tem papel preponderante (SHEN et al., 1991). A textura do tofu deve ser coesa, lisa e firme, mas não dura ou borrachenta. Devido ao sabor quase neutro deste produto, as propriedades de textura do tofu têm importante papel na qualidade e na aceitação pelo consumidor (LIM et al., 1990). Sua textura é principalmente influenciada pelo processo de fabricação e pelas características da cultivar utilizada, e a preferência quanto ao grau de dureza do tofu varia segundo o hábito cultural e o gosto pessoal do consumidor (CUI et al., 2004).

Sabendo que as cultivares de soja têm diferente potencial para produzir tofu e outros derivados, há interesse de se identificar e direcionar as cultivares mais adequadas para cada uso específico, tanto para a comercialização interna como exportação (EVANS; TSUKAMOTO; NIELSEN, 1997). Cultivares de soja que produzem tofu com alto rendimento, alto teor de proteínas e boas propriedades de textura são consideradas mais adequadas para obtenção do produto (CAI et al., 1997).

O conteúdo de proteína no grão é um fator que influencia o rendimento do tofu (CAI et al., 1997; POYSA; WOODROW, 2002; MIN; YU; ST. MARTIN, 2005). Lim et al. (1990) consideraram que o conteúdo de proteína do grão, isoladamente, não é suficiente para afetar o rendimento do tofu. Min, Yu e St. Martin (2005) observaram que o conteúdo de proteína do tofu estava mais intimamente relacionado com a proteína do extrato de soja do que com a proteína do grão, uma vez que a proteína presente no extrato é completamente coagulada na forma de tofu.

O tamanho dos grãos de soja pode interferir no rendimento do tofu. Shen et al. (1991) relataram que os grãos pequenos contêm maior área de casca por unidade de peso que os grãos grandes. Cui et al. (2004) também comentaram que os grãos maiores e esféricos geralmente apresentam relação superfície/volume mais favorável à extração dos sólidos solúveis que os pequenos, uma vez que isto reduz a proporção de cascas; por isto, os grãos maiores tendem a aumentar o rendimento do tofu. Poysa e Woodrow (2002) encontraram correlação positiva entre o tamanho do grão e o rendimento do tofu. No entanto, Lim et al. (1990) e Shen et al. (1991), estudando a produção de tofu a partir de nove cultivares de soja com grande variação de tamanho, não encontraram correlações entre tamanho de grãos e rendimento, mesmo tendo utilizado dois diferentes coagulantes, glucono-deltalactona (GDL) e sulfato de cálcio.

Vários pesquisadores, citados a seguir, buscaram desenvolver métodos para discriminar o desempenho comparativo de variedades e linhagens de soja a serem utilizadas na produção de tofu ou outros produtos de soja. Os métodos manuais, de laboratório, por dependerem mais da habilidade do manipulador, dificilmente fornecem resultados reprodutíveis, conforme relatado por Cai e Chang (1998). Estes mesmos autores, citando Murphy et al. (1997), sugerem que a avaliação do potencial de uma cultivar para produção de tofu requer uma escala de produção maior e automatizada. Hou, Chang e Shih (1997) afirmaram que os métodos em escala de laboratório, usando quantidades pequenas (80-150g) de grãos, geralmente são difíceis de reproduzir, devido aos complexos fatores envolvidos. Essas percepções foram confirmadas pelo estudo de Cai et al. (1997) que, comparando os dois tipos de métodos, mostraram que o automatizado era mais adequado para testar cultivares comerciais de soja para uso em processos industriais, enquanto o método de batelada, em menor escala, era útil para avaliação de cultivares e linhagens em desenvolvimento, quando a quantidade de grãos disponível é pequena. Evans, Tsukamoto e Nielsen (1997) testaram um método descontínuo comparativo com grande número de cultivares, observando diferenças significativas no rendimento do extrato e na eficiência de recuperação da proteína e de sólidos da soja no tofu, bem como em sua textura. Mullin et al. (2001) também procuraram 
desenvolver um procedimento rápido e reprodutível de obtenção de tofu tipo silken, para avaliação de cultivares de soja, tendo por objetivo padronizar um método para comparações interlaboratoriais.

Os testes sensoriais afetivos têm por objetivo obter a resposta pessoal (preferência ou aceitação) de consumidores ou consumidores potenciais sobre um determinado produto e os resultados obtidos são, geralmente, avaliados por análise de variância (ANOVA) univariada e teste de comparação de médias (MEILGAARD; CIVILLE; CARR, 2007). No entanto, o uso da média de aceitação pode encobrir a existência de categorias de consumidores com preferências opostas, quando as respostas de um grupo cancelam as de outro (OLIVEIRA et al., 2004). Dentre as técnicas utilizadas em testes de consumidor, destacase o Mapa de Preferência, que utiliza técnicas de estatística multivariada para fazer uma representação das diferenças de aceitação entre produtos, permitindo a identificação de cada indivíduo e suas preferências (CALEGUER; TOFFOLI; BENASSI, 2006). No Mapa de Preferência Interno, os resultados de aceitação dos consumidores geram um espaço onde os produtos são alocados (MEILGAARD; CIVILLE; CARR, 2007).

Neste trabalho, grãos de oito cultivares brasileiras de soja foram utilizados como matéria-prima para obtenção de tofu, por meio de um processo em escala de laboratório, previamente definido. Com o objetivo de avaliar o potencial das cultivares de soja para esta aplicação, foram determinadas algumas características físicas e químicas dos grãos e dos tofus, bem como o rendimento do processo e a aceitação sensorial dos produtos.

\section{Material e Métodos}

\section{Material}

Foram selecionadas para o experimento oito cultivares de soja com características diversificadas (CULTIVARES, 2005; CULTIVARES, 2008; CARRÃO-PANIZZI et al., 2009a; CARRÃOPANIZZI et al., 2009b):
BRS 155 - teor reduzido de inibidor de tripsina (1/3 do normal);

BRS 216 - grãos pequenos, alto teor de proteínas e de isoflavonas;

BRS 232 - grãos grandes;

BRS 257 - ausência de enzimas lipoxigenases $\left(\mathrm{L}_{1}\right.$, $\mathrm{L}_{2} \mathrm{e}_{3}$ );

BRS $258 \neg$-descendente da cultivar BR 36, bastante usada na alimentação humana;

BRS 262 - hilo preto e alto teor de isoflavonas;

BRS 267 - grãos de tamanho acima da média e sabor considerado suave;

BRS MG 790A - casca alaranjada e sabor considerado bom.

As cultivares desenvolvidas no programa de melhoramento genético da Embrapa Soja, Londrina/ PR, foram obtidas na safra 2007/2008 em Londrina e Ponta Grossa, com exceção da BRS MG 790A, proveniente do Triângulo Mineiro. As amostras foram mantidas em câmara fria $\left(10^{\circ} \mathrm{C}\right.$ e $68 \%$ UR) até utilização.

\section{Métodos}

Composição centesimal de grãos e tofu de cultivares de soja

Os conteúdos de umidade, proteínas, lipídios e cinzas foram determinados em amostras de grãos e tofu por métodos padrão (AOAC, 1995), com três repetições. $\mathrm{O}$ teor de nitrogênio foi determinado por Kjeldahl, usando um equipamento automático (FOSS, Tecator 2400, Dinamarca) para destilação e titulação; o fator 6,25 foi empregado para o cálculo da proteína. $O$ conteúdo de carboidratos foi calculado por diferença.

\section{Tamanho de grãos}

O tamanho dos grãos de soja foi expresso em termos de massa e de dimensões. 
Para avaliar o peso de 100 grãos: amostras de 100 grãos foram constituídas por duas porções de 50 grãos, escolhidos com o auxílio de uma placa de madeira contendo cavidades em formato de meia esfera, onde os grãos de soja de quaisquer tamanhos podem se encaixar. As amostras, com nove repetições para cada cultivar, foram pesadas em balança semianalítica (Bel Engineering, Mark 2200, Brasil). Os resultados foram expressos em g/100 grãos. Os grãos foram classificados de acordo com o seguinte critério: grandes $\geq 20 \mathrm{~g}$, pequenos $\leq 10 \mathrm{~g}$ e intermediários entre 10 e 20 g/100 grãos (YOKOMIZO; DUARTE.; VELLO, 2000).

Considerando-se que o grão de soja tem o formato aproximado de uma esfera, o diâmetro (d) dos grãos foi medido com auxílio de um paquímetro digital (Mitutoyo, modelo CD-S6"C, Japão), ajustado na região mediana do grão, no eixo transversal que passa pelo hilo. Foram realizadas 20 repetições para cada cultivar. Com o valor do raio ( $r)$ da esfera $(r=d / 2$, em $\mathrm{mm}$ ), foram calculados o volume aproximado dos grãos $\left(V=4 / 3 \pi r^{3}\right.$, em $\left.\mathrm{mm}^{3}\right)$ e sua área superficial (A $=4 \pi \mathrm{r}^{2}$, em $\mathrm{mm}^{2}$ ), para cada cultivar.

\section{Determinação da quantidade de cascas nos grãos}

Amostras de grãos de soja foram desidratadas em liofilizador (Liobrás, Liotop L101, Brasil), para permitir a retirada das cascas. Cada alíquota foi pesada em balança analítica (Mettler Toledo, modelo AT-201, Suíça) e embalada em filme plástico (aproximadamente $3 \mathrm{~cm} \times 3 \mathrm{~cm}$ ), perfurado com auxílio de uma agulha. As amostras das oito cultivares foram acondicionadas em prato de inox posicionado na bandeja central do liofilizador e submetidas a $24 \mathrm{~h}$ de desidratação $\left(\mathrm{T}=-55^{\circ} \mathrm{C}\right.$, pressão de $\left.60-80 \mu \mathrm{Hg}\right)$. As amostras foram retiradas do liofilizador e colocadas em um dessecador. Cada uma das amostras foi retirada do invólucro plástico, o peso de seus grãos registrado e, com auxílio de luvas e pinça, os grãos foram descascados um a um, pesando-se posteriormente as cascas. A quantidade de cascas foi expressa em $\mathrm{g}$ casca/100 g grãos. Foram realizadas nove repetições do processo. A relação entre massa e área superficial das cascas dos grãos de soja das cultivares, expressa em $\mathrm{mg} / \mathrm{cm}^{2}$, foi calculada por: Relação massa/área $=10$ x Conteúdo cascas x Peso 100 grãos x 1/Área superficial (dados das Tabelas 2 e 3).

\section{Processamento do tofu}

A produção de tofu a partir dos grãos de soja foi realizada usando sulfato de cálcio diidratado $\left(\mathrm{CaSO}_{4} \cdot 2 \mathrm{H}_{2} \mathrm{O}\right.$, marca Wako, Japão). As condições de processamento foram previamente definidas por Benassi, Yamashita e Prudencio (2011), no qual utilizaram apenas a cultivar BRS 267. As principais etapas do processo são: maceração de $150 \mathrm{~g}$ de grãos em $500 \mathrm{~mL}$ de água à temperatura ambiente por $16 \mathrm{~h}$; drenagem; trituração com cerca de $1 \mathrm{~L}$ de água, a quente $\left(90^{\circ} \mathrm{C}\right)$, em liquidificador doméstico (Arno, Performa Magiclean, Brasil) na velocidade 3 por 3 min; filtração à vácuo (bomba Vacuubrand, $\mathrm{MZ} 2 \mathrm{C}$, Alemanha), por $10 \mathrm{~min}$, em funil de Buchner forrado com tecido fino de nylon ("tunil"); aquecimento do extrato de soja em recipiente tampado, sobre o fogo direto, até a fervura, seguido de mais $10 \mathrm{~min}$, em banho-maria em ebulição; homogeneização (usando bastão de vidro) de $760 \mathrm{~mL}$ de extrato com solução de $\mathrm{CaSO}_{4}$ (3 g em 40 mLágua morna), adicionada de uma única vez; coagulação por $10 \mathrm{~min}$ a $75-76^{\circ} \mathrm{C}$; corte do coágulo com auxílio de uma lira de inox; colocação do coágulo em forma plástica de aproximadamente $500 \mathrm{~mL}$ perfurada e forrada com "tunil"; aplicação de uma força de 9,8 $\mathrm{N}$ sobre a superfície, produzindo uma pressão de $1,15 \mathrm{KPa}$, por $30 \mathrm{~min}$; retirada da forma e conservação em geladeira, em recipiente fechado, contendo água até cobrir o tofu. Três repetições do procedimento foram realizadas para cada cultivar, em ordem aleatória.

\section{Absorção de água pelos grãos de soja durante a maceração}

A diferença entre a massa de grãos macerados por $16 \mathrm{~h}$ (item 2.2.4) e a massa inicial de grãos 
forneceu a absorção de água, expressa em g de água absorvida/100 g de grãos.

\section{Rendimento em tofu}

A massa de cada tofu foi relacionada à massa inicial de grãos, e o rendimento expresso em termos de $g$ tofu/100 g de grãos.

\section{Dureza dos tofus}

Foi determinada em texturômetro TA.XT2i (marca Stable Micro Systems, Inglaterra), utilizando sensor cilíndrico de alumínio de 35mm de diâmetro (P35), para comprimir amostras cilíndricas de tofu, $(3 \mathrm{~cm}$ diâmetro x 2,2cm altura) até $73 \%$ de deformação. As velocidades de pré-teste, teste e pós-teste foram, respectivamente, de 2,0, 2,0 e 4,0 mm/s (ROSSET, 2007). Foram realizadas seis repetições para cada tofu e os resultados foram expressos em Newton (N).

\section{Análise sensorial}

A análise sensorial foi conduzida após aprovação do projeto pelo Comitê de Ética em Pesquisa envolvendo Seres Humanos da Universidade Estadual de Londrina (parecer No. 216/07).

Os tofus produzidos foram avaliados, em ordem aleatória, por um teste de Aceitação de Atributos, em duas sessões, com quatro amostras de tofu em cada uma delas. As amostras, codificadas com números de três algarismos, foram apresentadas monadicamente aos julgadores, que avaliaram os atributos sabor, textura e aceitação global, utilizando escala hedônica estruturada de nove pontos, ancorada nos extremos e no meio, onde: $9=$ gostou extremamente, $5=$ nem gostou, nem desgostou, $1=$ desgostou extremamente.

A equipe de julgadores foi formada por 71 indivíduos não treinados (37 homens e 34 mulheres), com idades entre 16 e 54 anos (sendo mais numerosa a faixa de 21 a 30 anos, com 44\%) e grau de instrução variado $(50 \%$ com nível universitário e pós-graduação). Para participação no teste, não era necessário que o indivíduo fosse um consumidor habitual de tofu, porém $83 \%$ afirmaram consumir produtos de soja, com diferente frequência (destes, as respostas "esporadicamente" e "frequentemente" somaram 68\%); para os $17 \%$ que declararam não consumir produtos de soja, a idéia de introduzir a soja e seus derivados na alimentação foi considerada boa (41\%) ou muito boa (24\%). Entre os alimentos de soja que já consumiu ou consome, o tofu foi indicado por $42 \%$ do total de julgadores.

\section{Delineamento experimental e análise estatística dos dados}

O experimento foi conduzido de acordo com delineamento inteiramente casualizado. Para análise sensorial, o delineamento foi de blocos completos casualizados. Os resultados foram tratados por ANOVA e teste de comparação de médias (Tukey, $\mathrm{p} \leq 0,05)$, utilizando o programa STATISTICA versão 6.0 (STATSOFT, 2001). Para avaliação do Mapa de Preferência Interno, empregou-se o programa SENSTOOLS versão 2.3 (OP \& PRODUCT RESEARCH, 1998).

\section{Resultados e Discussão}

Os valores encontrados para a composição centesimal dos grãos das cultivares de soja (Tabela 1) estão dentro das faixas citadas por outros autores (LIM et al., 1990; SHEN et al., 1991; CAI et al., 1997; CAI; CHANG, 1998; POYSA; WOODROW, 2002; POYSA; WOODROW; YU, 2006; ROSSET, 2007; DA SILVA, 2009). A umidade dos grãos, entre 9 e $11 \%$, está na faixa recomendada para conservação dos mesmos. A diferença entre o maior e o menor teor protéico foi de apenas $4 \mathrm{~g} / 100 \mathrm{~g}$ de grãos (cerca de $11 \%)$, porém, significativa $(\mathrm{p} \leq 0,05)$. O teor de lipídios variou entre $19 \mathrm{~g} / 100 \mathrm{~g}$ (BRSMG 790A) e $24 \mathrm{~g} / 100 \mathrm{~g}$ (BRS 258) e o teor de cinzas, entre $6 \mathrm{~g} / 100 \mathrm{~g}$ (BRS 216) e $8 \mathrm{~g} / 100 \mathrm{~g}$ (BRS 267). 
Tabela 1. Composição centesimal dos grãos de soja.

\begin{tabular}{lccccc}
\hline Cultivar & Umidade $^{\mathbf{1}}$ & Proteína $^{\mathbf{2}}$ & Lipídios $^{\mathbf{2}}$ & Cinzas $^{\mathbf{2}}$ & Carboidratos $^{\mathbf{2}}$ \\
\hline BRS 155 & $10,82 \pm 0,54^{\mathrm{a}}$ & $39,07 \pm 0,68^{\mathrm{ab}}$ & $22,62 \pm 1,02^{\mathrm{ab}}$ & $6,36 \pm 0,27^{\mathrm{bc}}$ & 31,95 \\
BRS 216 & $10,51 \pm 0,08^{\mathrm{a}}$ & $39,54 \pm 0,95^{\mathrm{ab}}$ & $20,68 \pm 1,08^{\mathrm{c}}$ & $5,89 \pm 0,25^{\mathrm{d}}$ & 33,89 \\
BRS 232 & $9,81 \pm 0,25^{\mathrm{b}}$ & $39,61 \pm 0,46^{\mathrm{a}}$ & $21,25 \pm 1,04^{\mathrm{bc}}$ & $6,63 \pm 0,25^{\mathrm{b}}$ & 32,51 \\
BRS 257 & $9,87 \pm 0,13^{\mathrm{b}}$ & $38,86 \pm 0,61^{\mathrm{ab}}$ & $20,19 \pm 0,97^{\mathrm{cd}}$ & $6,04 \pm 0,39^{\mathrm{cd}}$ & 34,91 \\
BRS 258 & $9,35 \pm 0,43^{\mathrm{c}}$ & $38,62 \pm 1,37^{\mathrm{ab}}$ & $23,77 \pm 0,87^{\mathrm{a}}$ & $5,95 \pm 0,44^{\mathrm{cd}}$ & 31,66 \\
BRS 262 & $9,62 \pm 0,11^{\mathrm{bc}}$ & $35,46 \pm 0,46^{\mathrm{c}}$ & $22,56 \pm 1,80^{\mathrm{ab}}$ & $6,62 \pm 0,40^{\mathrm{b}}$ & 35,36 \\
BRS 267 & $10,01 \pm 0,39^{\mathrm{b}}$ & $38,47 \pm 0,32^{\mathrm{b}}$ & $18,55 \pm 1,42^{\mathrm{d}}$ & $7,69 \pm 0,16^{\mathrm{a}}$ & 35,29 \\
BRSMG 790A & $8,76 \pm 0,17^{\mathrm{d}}$ & $36,34 \pm 0,38^{\mathrm{c}}$ & $22,84 \pm 1,14^{\mathrm{ab}}$ & $6,71 \pm 0,16^{\mathrm{b}}$ & 34,11 \\
\hline
\end{tabular}

Letras diferentes na mesma coluna indicam diferença significativa (Tukey, $\mathrm{p} \leq 0,05$ )

Média de 3 determinações \pm desvio padrão

(1) resultados expressos em $\mathrm{g} / 100 \mathrm{~g}$ matéria integral

(2) resultados expressos em $\mathrm{g} / 100 \mathrm{~g}$ matéria seca.

O tamanho dos grãos das cultivares de soja, expresso em termos de massa e de dimensões, está na Tabela 2. A BRS 232 apresenta grãos grandes e a BRS 216, pequenos, enquanto as demais cultivares possuem valores intermediários. Apenas BRS $155 \mathrm{e}$ BRSMG 790A apresentaram grãos de peso idêntico. Segundo Cai et al. (1997), a indústria de tofu prefere grãos de tamanho grande. A correlação entre o peso e as dimensões dos grãos (expresso em diâmetro, área superficial ou volume) foi de $0,98(\mathrm{p} \leq 0,01)$, ou seja, todas estas grandezas representam igualmente o tamanho do grão. Assim, as cultivares podem ser ordenadas, do maior para o menor tamanho de grãos, da seguinte maneira: BRS 232, BRS 267, BRS 258, BRSMG 790A, BRS 155, BRS 257, BRS 262 e BRS 216.

Tabela 2. Peso e dimensões de grãos de cultivares de soja.

\begin{tabular}{lllll}
\hline Cultivar & Peso 100 grãos $^{\mathbf{1}}$ & \multicolumn{1}{c}{ Diâmetro $^{2}$} & Área superficial $^{\mathbf{3}}$ & \multicolumn{1}{c}{ Volume $^{4}$} \\
\hline BRS 155 & $17,04 \pm 0,21 \mathrm{~d}$ & $6,63 \pm 0,29 \mathrm{bc}$ & $138,37 \pm 12,15 \mathrm{~d}$ & $153,48 \pm 20,08 \mathrm{~d}$ \\
BRS 216 & $10,83 \pm 0,17 \mathrm{~g}$ & $5,71 \pm 0,32 \mathrm{~d}$ & $102,89 \pm 11,57 \mathrm{f}$ & $98,58 \pm 16,80 \mathrm{f}$ \\
BRS 232 & $22,16 \pm 0,55 \mathrm{a}$ & $7,35 \pm 0,49 \mathrm{a}$ & $170,48 \pm 22,54 \mathrm{a}$ & $210,62 \pm 41,38 \mathrm{a}$ \\
BRS 257 & $14,80 \pm 0,19 \mathrm{e}$ & $6,47 \pm 0,25 \mathrm{~cd}$ & $131,69 \pm 9,98 \mathrm{de}$ & $142,40 \pm 16,03 \mathrm{de}$ \\
BRS 258 & $18,32 \pm 0,43 \mathrm{c}$ & $7,03 \pm 0,29 \mathrm{ab}$ & $155,62 \pm 12,66 \mathrm{bc}$ & $182,97 \pm 22,19 \mathrm{bc}$ \\
BRS 262 & $14,01 \pm 0,35 \mathrm{f}$ & $6,13 \pm 0,34 \mathrm{~cd}$ & $118,42 \pm 13,20 \mathrm{e}$ & $121,71 \pm 20,24 \mathrm{ef}$ \\
BRS 267 & $19,11 \pm 0,44 \mathrm{~b}$ & $7,15 \pm 0,47 \mathrm{ab}$ & $161,34 \pm 21,36 \mathrm{ab}$ & $193,90 \pm 38,59 \mathrm{ab}$ \\
BRSMG 790A & $16,70 \pm 0,21 \mathrm{~d}$ & $6,77 \pm 0,25 \mathrm{~b}$ & $144,22 \pm 10,68 \mathrm{~cd}$ & $163,18 \pm 17,98 \mathrm{~cd}$ \\
\hline
\end{tabular}

Letras diferentes na mesma coluna indicam diferença significativa (Tukey, $\mathrm{p} \leq 0,05$ )

(1) resultados expressos em g; média de 9 repetições \pm desvio padrão; grãos pequenos $\leq 10 \mathrm{~g} / 100$ grãos e grãos grandes $\geq 20 \mathrm{~g} / 100$ grãos $^{(2)}$ resultados expressos em $\mathrm{mm}$ (para um grão); média de 20 repetições \pm desvio padrão ${ }^{(3)}$ resultados expressos em $\mathrm{mm}^{2}$ (para um grão); média de 20 repetições \pm desvio padrão ${ }^{(4)}$ resultados expressos em $\mathrm{mm}^{3}$ (para um grão); média de 20 repetições \pm desvio padrão. 
Tabela 3. Conteúdo de cascas nos grãos das cultivares de soja e relação entre massa e área das cascas.

\begin{tabular}{lcc}
\hline Cultivar & Conteúdo de cascas $^{1}$ & Relação massa/área $^{2}$ \\
\hline BRS 155 & $6,28 \pm 0,38 \mathrm{f}$ & 7,67 \\
BRS 216 & $7,69 \pm 0,51 \mathrm{bc}$ & 9,25 \\
BRS 232 & $6,47 \pm 0,18 \mathrm{ef}$ & 8,84 \\
BRS 257 & $8,02 \pm 0,57 \mathrm{ab}$ & 8,85 \\
BRS 258 & $7,08 \pm 0,27 \mathrm{cde}$ & 8,48 \\
BRS 262 & $8,34 \pm 0,63 \mathrm{a}$ & 9,87 \\
BRS 267 & $7,16 \pm 0,38 \mathrm{~cd}$ & 8,50 \\
BRSMG 790A & $6,84 \pm 0,24 \mathrm{def}$ & 8,22 \\
\hline
\end{tabular}

Letras diferentes na mesma coluna indicam diferença significativa (Tukey, $\mathrm{p} \leq 0,05$ )

(1) resultados expressos em $\mathrm{g}$ de casca/100 $\mathrm{g}$ de grãos; média de 9 repetições \pm desvio padrão

(2) resultados expressos em $\mathrm{mg} / \mathrm{cm}^{2}$ de cascas; valores calculados.

Por ser muito fina, a espessura das cascas de soja é difícil de ser medida com precisão. Para contornar este problema, sem recorrer a métodos de microscopia, foi calculada a relação entre massa e área superficial de cascas. A partir destes valores, foi possível supor que a espessura (ou porosidade ou densidade da casca) variou entre as cultivares, uma vez que cada $\mathrm{cm} 2$ de casca pode pesar desde $7,7 \mathrm{mg}$ para BRS 155 até 9,9 mg para BRS 262.

Mullin et al. (2001) consideraram que a determinação da composição química dos grãos e suas características físicas não são suficientes para julgar a adequação de uma cultivar de soja para produção de tofu. Os autores afirmam que é necessário obter o produto e determinar seu rendimento, composição, teor de sólidos e textura.

O processo para obtenção do tofu, inicialmente definido a partir da cultivar BRS 267, mostrou-se adequado para ser usado com as demais cultivares. Perdas da ordem de $6 \pm 1 \%$ ocorreram na etapa de filtração, quando foram separados o extrato e o resíduo de soja. Devido a esta variabilidade (aproximadamente 17\%), é difícil alcançar reprodutibilidade no processamento realizado em laboratório, embora se tenham padronizado ao máximo as condições para produção de tofu. Este fato também foi observado por Cai et al. (1997), ao comparar dois tipos de processos, um automatizado e outro em batelada, sem o uso de equipamentos apropriados e/ou automáticos.

As medidas registradas durante 0 processamento do tofu possibilitaram calcular a quantidade de água absorvida pelos grãos durante a maceração e o rendimento em tofu, para cada uma das cultivares, cujos valores estão na Tabela 4.

O rendimento em tofu observado para as diferentes cultivares de soja foi semelhante $(\mathrm{p}>0,05)$. Porém, é possível notar que, entre o maior e o menor rendimento, há uma diferença de aproximadamente $10 \%$, que provavelmente não foi significativa devido à variabilidade ocorrida entre as três repetições do processamento. 
Tabela 4. Rendimento em tofu e água absorvida na maceração dos grãos de cultivares de soja.

\begin{tabular}{lcc}
\hline Cultivar & $\begin{array}{c}\text { Rendimento } \\
\text { em tofu }^{\mathbf{1}}\end{array}$ & $\begin{array}{c}\text { \% água } \\
\text { absorvida }^{\mathbf{2}}\end{array}$ \\
\hline BRS 155 & $265,31 \pm 10,30 \mathrm{a}$ & $120,55 \pm 0,33 \mathrm{~d}$ \\
BRS 216 & $264,37 \pm 9,22 \mathrm{a}$ & $127,81 \pm 0,34 \mathrm{~cd}$ \\
BRS 232 & $268,38 \pm 9,23 \mathrm{a}$ & $126,42 \pm 7,26 \mathrm{~cd}$ \\
BRS 257 & $263,05 \pm 5,80 \mathrm{a}$ & $130,17 \pm 1,29 \mathrm{bc}$ \\
BRS 258 & $273,47 \pm 12,78 \mathrm{a}$ & $127,60 \pm 0,89 \mathrm{~cd}$ \\
BRS 262 & $264,46 \pm 11,72 \mathrm{a}$ & $132,42 \pm 1,37 \mathrm{abc}$ \\
BRS 267 & $288,58 \pm 21,43 \mathrm{a}$ & $135,41 \pm 0,35 \mathrm{ab}$ \\
BRSMG 790A & $281,98 \pm 16,64 \mathrm{a}$ & $139,99 \pm 0,66 \mathrm{a}$ \\
\hline
\end{tabular}

Letras diferentes na mesma coluna indicam diferença significativa (Tukey, $p \leq 0,05$ )

(1) resultados expressos em $\mathrm{g}$ tofu/100 g grãos; média de 3 repetições \pm desvio padrão

(2) resultados expressos em g água/100 g grãos; média de 3 repetições \pm desvio padrão.

A literatura relata efeitos de diferentes variáveis sobre o rendimento. Cui et al. (2004) relataram correlação positiva do rendimento com o teor de proteína e com o peso de 100 grãos, ressaltando, porém, que os cuidados na pós-colheita e condicionamento dos grãos podem ter maior influência que a própria variação genética. Min, Yu e St. Martin (2005) também observaram influência do teor de proteínas do grão sobre o rendimento do tofu e sugeriram que a determinação de proteínas no grão pode ser usada para predizer o rendimento e o conteúdo protéico do tofu. Lim et al. (1990) e Shen et al. (1991) relataram que o rendimento em tofu não foi significativamente afetado pelo tamanho ou teor de proteínas dos grãos; porém, em seus estudos há modelos preditivos para o rendimento do tofu, nos quais a variável proteína no grão está presente, evidenciando que ela é importante, apesar de não ser a única responsável pelo rendimento.

$\mathrm{Na}$ etapa de maceração dos grãos para o preparo de tofu, foi notado que as cultivares diferiram quanto à capacidade de absorção de água: as cultivares BRS 155, BRS 216 e BRS 258 absorveram menor quantidade, enquanto BRS 267 e BRSMG 790A absorveram maior quantidade de água. Supondo que a capacidade de absorção de água poderia influenciar as características do tofu obtido, investigaram-se as correlações existentes, tendo sido observada correlação inversa $(\mathrm{r}=$ $0,75, \mathrm{p} \leq 0,05$ ) com a dureza, ou seja, os grãos que absorveram mais água na maceração foram os que, posteriormente, resultaram em tofu com menor dureza.

Poysa e Woodrow (2002) não encontraram correlação da absorção de água dos grãos com o rendimento do tofu, nem com outro atributo de qualidade, mencionando tanto trabalhos em concordância (WANG et al., 1983) como conflitantes (BHARDWAJ et al., 1999) com seus resultados.

A composição centesimal dos tofus, na Tabela 5 , foi similar à encontrada por outros autores (LIM et al., 1990; SHEN et al., 1991; CAI et al., 1997; CAI; CHANG, 1998; POYSA; WOODROW, 2002; POYSA; WOODROW; YU, 2006; ROSSET, 2007). Apenas o conteúdo de lipídios, variando entre 23 e $30 \mathrm{~g} / 100 \mathrm{~g}$, apresentou-se superior aos valores da literatura (entre 16,5 e 21,5 g/100g). Provavelmente, as condições de processamento utilizadas promoveram uma remoção extensiva dos lipídios dos grãos, os quais foram transferidos ao extrato de soja e, posteriormente, ao tofu. A correlação entre a composição centesimal dos grãos de soja e dos tofus (Tabelas 1 e 5) foi significativa apenas para o conteúdo de proteína $(\mathrm{r}=0,83$, $\mathrm{p} \leq 0,05)$.

Os valores de dureza instrumental e as notas de aceitação atribuídas pelos julgadores aos tofus segundo os atributos sabor, textura e aceitação global, estão na Tabela 6. A dureza dos tofus variou de 2,3N (BRS 267) a 3,8N (BRS 216) e apresentou correlação significativa com a textura sensorial $(\mathrm{r}=0,80, \mathrm{p} \leq 0,05)$ e com o conteúdo de proteínas, tanto do tofu $(\mathrm{r}=0,75, \mathrm{p} \leq 0,05)$ quanto do grão $(\mathrm{r}=0,79, \mathrm{p} \leq 0,05)$, este último em concordância com o relatado por Evans, Tsukamoto e Nielsen (1997). 
Tabela 5. Composição centesimal de tofus de cultivares de soja.

\begin{tabular}{lccccc}
\hline Cultivar & Umidade $^{1}$ & Proteína $^{2}$ & Lipídios $^{\mathbf{2}}$ & Cinzas $^{2}$ & Carboidratos $^{\mathbf{2}}$ \\
\hline BRS 155 & $86,98 \pm 1,13^{\text {ab }}$ & $52,80 \pm 1,00^{\text {bc }}$ & $23,30 \pm 1,79^{\text {cd }}$ & $5,63 \pm 0,69^{\mathrm{c}}$ & 18,27 \\
BRS 216 & $86,54 \pm 0,85^{\mathrm{b}}$ & $56,66 \pm 1,36^{\mathrm{a}}$ & $23,10 \pm 2,79^{\mathrm{d}}$ & $5,46 \pm 0,70^{\mathrm{c}}$ & 14,78 \\
BRS 232 & $86,51 \pm 1,02^{\mathrm{b}}$ & $54,04 \pm 2,04^{\mathrm{b}}$ & $27,61 \pm 1,31^{\mathrm{ab}}$ & $6,02 \pm 0,43^{\mathrm{abc}}$ & 12,33 \\
BRS 257 & $86,23 \pm 0,55^{\mathrm{b}}$ & $52,79 \pm 0,71^{\mathrm{bc}}$ & $27,84 \pm 1,37^{\mathrm{ab}}$ & $6,51 \pm 0,36^{\mathrm{ab}}$ & 12,86 \\
BRS 258 & $86,29 \pm 0,83^{\mathrm{b}}$ & $51,71 \pm 0,54^{\mathrm{cd}}$ & $25,75 \pm 1,18^{\mathrm{bc}}$ & $5,89 \pm 0,38^{\mathrm{bc}}$ & 16,65 \\
BRS 262 & $86,06 \pm 1,16^{\mathrm{b}}$ & $50,04 \pm 1,05^{\mathrm{e}}$ & $28,91 \pm 1,16^{\mathrm{a}}$ & $6,60 \pm 0,22^{\mathrm{a}}$ & 14,45 \\
BRS 267 & $88,04 \pm 0,42^{\mathrm{a}}$ & $53,36 \pm 0,46^{\mathrm{bc}}$ & $24,31 \pm 2,06^{\mathrm{cd}}$ & $6,58 \pm 0,21^{\mathrm{a}}$ & 15,75 \\
BRSMG 790 & $86,84 \pm 0,29^{\mathrm{ab}}$ & $50,60 \pm 0,88^{\mathrm{de}}$ & $30,02 \pm 1,55^{\mathrm{a}}$ & $5,47 \pm 0,37^{\mathrm{c}}$ & 13,91 \\
\hline
\end{tabular}

Letras diferentes na mesma coluna indicam diferença significativa (Tukey, $\mathrm{p} \leq 0,05$ )

Média de três determinações \pm desvio padrão

(1) resultados expressos em $\mathrm{g} / 100 \mathrm{~g}$ matéria integral

${ }^{(2)}$ resultados expressos em $\mathrm{g} / 100 \mathrm{~g}$ matéria seca.

Quanto ao sabor, os tofus BRS 257 e 216 foram mais apreciados (66 e 68\% de aprovação, respectivamente) que o $\mathrm{BRS} 267 \quad(44 \%$ de aprovação). A textura do tofu BRS 216 foi mais aceita que a do BRSMG 790A (respectivamente, 75 e 55\% de aprovação), enquanto os demais apresentaram valores intermediários, não diferindo entre si. $\mathrm{Na}$ aceitação global, observou-se que os tofus das diferentes cultivares foram igualmente aceitos, obtendo, numa escala de 1 a 9, a média 5,8 para todos os produtos. Deve-se considerar, no entanto que, para participar do teste, não havia a exigência de que o julgador fosse um consumidor freqüente de tofu, o que pode ter ocasionado resultados semelhantes e próximos da indiferença (nota 5) para todas as amostras.

Tabela 6. Valores de dureza instrumental e notas hedônicas de atributos sensoriais de tofus de cultivares de soja.

\begin{tabular}{|c|c|c|c|c|c|c|c|}
\hline \multirow[t]{3}{*}{ Tofu } & \multirow{3}{*}{$\begin{array}{c}\text { Dureza } \\
\text { Instrumental }^{1}\end{array}$} & \multicolumn{6}{|c|}{ Aceitação } \\
\hline & & \multicolumn{2}{|c|}{ Sabor } & \multicolumn{2}{|c|}{ Textura } & \multicolumn{2}{|c|}{ Global } \\
\hline & & média $^{2}$ & aprovação $^{3}$ & média $^{2}$ & aprovação $^{3}$ & média $^{2}$ & aprovação $^{3}$ \\
\hline BRS 155 & $3,4 \pm 0,7^{\mathrm{abc}}$ & $5,6 \pm 2,3^{\mathrm{ab}}$ & 55 & $6,2 \pm 1,8^{\mathrm{ab}}$ & 65 & $5,7 \pm 2,1^{\mathrm{a}}$ & 54 \\
\hline BRS 216 & $3,8 \pm 0,9^{\mathrm{a}}$ & $6,2 \pm 1,9^{a}$ & 68 & $6,4 \pm 1,6^{\mathrm{a}}$ & 75 & $6,2 \pm 1,5^{\mathrm{a}}$ & 70 \\
\hline BRS 232 & $3,6 \pm 0,5^{\mathrm{ab}}$ & $5,9 \pm 2,0^{\mathrm{ab}}$ & 59 & $5,9 \pm 2,1^{\mathrm{ab}}$ & 56 & $5,8 \pm 1,8^{\mathrm{a}}$ & 59 \\
\hline BRS 257 & $3,1 \pm 0,3^{\mathrm{bc}}$ & $6,2 \pm 2,1^{\mathrm{a}}$ & 66 & $5,9 \pm 1,9^{\mathrm{ab}}$ & 59 & $5,9 \pm 2,1^{\mathrm{a}}$ & 63 \\
\hline BRS 258 & $3,0 \pm 0,6^{\mathrm{cd}}$ & $5,6 \pm 2,0^{\mathrm{ab}}$ & 54 & $6,0 \pm 2,1^{\mathrm{ab}}$ & 63 & $5,6 \pm 2,0^{\mathrm{a}}$ & 52 \\
\hline BRS 262 & $2,4 \pm 0,5^{\mathrm{e}}$ & $6,0 \pm 1,9^{\mathrm{ab}}$ & 62 & $5,8 \pm 2,1^{\mathrm{ab}}$ & 56 & $5,7 \pm 1,9^{\mathrm{a}}$ & 54 \\
\hline BRS 267 & $2,3 \pm 0,5^{\mathrm{e}}$ & $5,3 \pm 2,0^{\mathrm{b}}$ & 44 & $5,8 \pm 2,0^{\mathrm{ab}}$ & 56 & $5,4 \pm 2,0^{\mathrm{a}}$ & 49 \\
\hline BRS MG 790A & $2,5 \pm 0,3^{\mathrm{de}}$ & $5,7 \pm 2,1^{\mathrm{ab}}$ & 51 & $5,6 \pm 2,2^{b}$ & 55 & $5,7 \pm 1,8^{a}$ & 52 \\
\hline
\end{tabular}

Letras diferentes na mesma coluna indicam diferença significativa (Tukey, $\mathrm{p} \leq 0,05$ )

(1) resultados expressos em $\mathrm{N}$; média de 6 repetições \pm desvio padrão

(2) notas hedônicas segundo a escala: 1=desgostou extremamente, 5=nem gostou, nem desgostou, 9=gostou extremamente; média de 71 julgadores \pm desvio padrão

(3) resultados expressos em porcentagem de notas $\geq 6$. 
A partir dos resultados do teste de aceitação, também foi construído o Mapa de Preferência Interno para cada um dos atributos sensoriais avaliados (Figura 1). Assim, foi possível observar, em relação aos três atributos, a distribuição dos julgadores em três grupos distintos. $\mathrm{O}$ Grupo 1 foi formado pelos indivíduos que gostaram dos tofus produzidos a partir de BRS 267 e BRSMG 790A, enquanto que no Grupo 2 foram alocados os provadores que preferiram os produtos BRS 155 e 258; e no Grupo 3, de BRS 216, 232, 257 e 262.

$\mathrm{Na}$ preferência quanto ao sabor (Figura 1A), os consumidores do Grupo 1 ( $\mathrm{N}=16$ indivíduos) alocaram-se em área oposta do mapa aos do Grupo $2(\mathrm{~N}=20)$, indicando apreciação ou preferência por tipos diferentes de tofu. Quanto aos consumidores do Grupo 3, uma parte deles se aproximou mais de um ou outro dos grupos anteriores. O sabor do tofu BRS 257 foi preferido por $45 \%$ dos consumidores $(\mathrm{N}=32) \mathrm{e}$ a principal característica desta cultivar é a ausência de enzimas lipoxigenases.

$\mathrm{Na}$ grande maioria das cultivares, coexistem nos grãos ácidos graxos poliinsaturados (particularmente o linolênico) e enzimas lipoxigenases, que catalisam a oxidação destes ácidos em aldeídos, cetonas e alcoóis, os quais podem ter aroma ou sabor indesejável (ROBINSON et al., 1995). Entre estes compostos, o n-hexanal está presente em maior quantidade e é o que mais contribui para o aparecimento do sabor beany associado com vários produtos à base de soja (LIU, 1999; TORRES-PENARANDA et al., 1998). O sabor beany costuma desagradar à população ocidental e é descrito como assemelhado ao de feijão cru, de grama ou mesmo de ranço. A ação das lipoxigenases sobre os lipídios é favorecida pela maceração a frio utilizada no processamento do tofu. O desenvolvimento de cultivares sem enzimas lipoxigenases é uma das alternativas mais viáveis para obter derivados de soja com sabor mais suave (LIU, 1999).

Figura 1. Mapas de Preferência Interno para Sabor (A), Textura (B) e Aceitação global (C) de tofus obtidos de diferentes cultivares de soja.
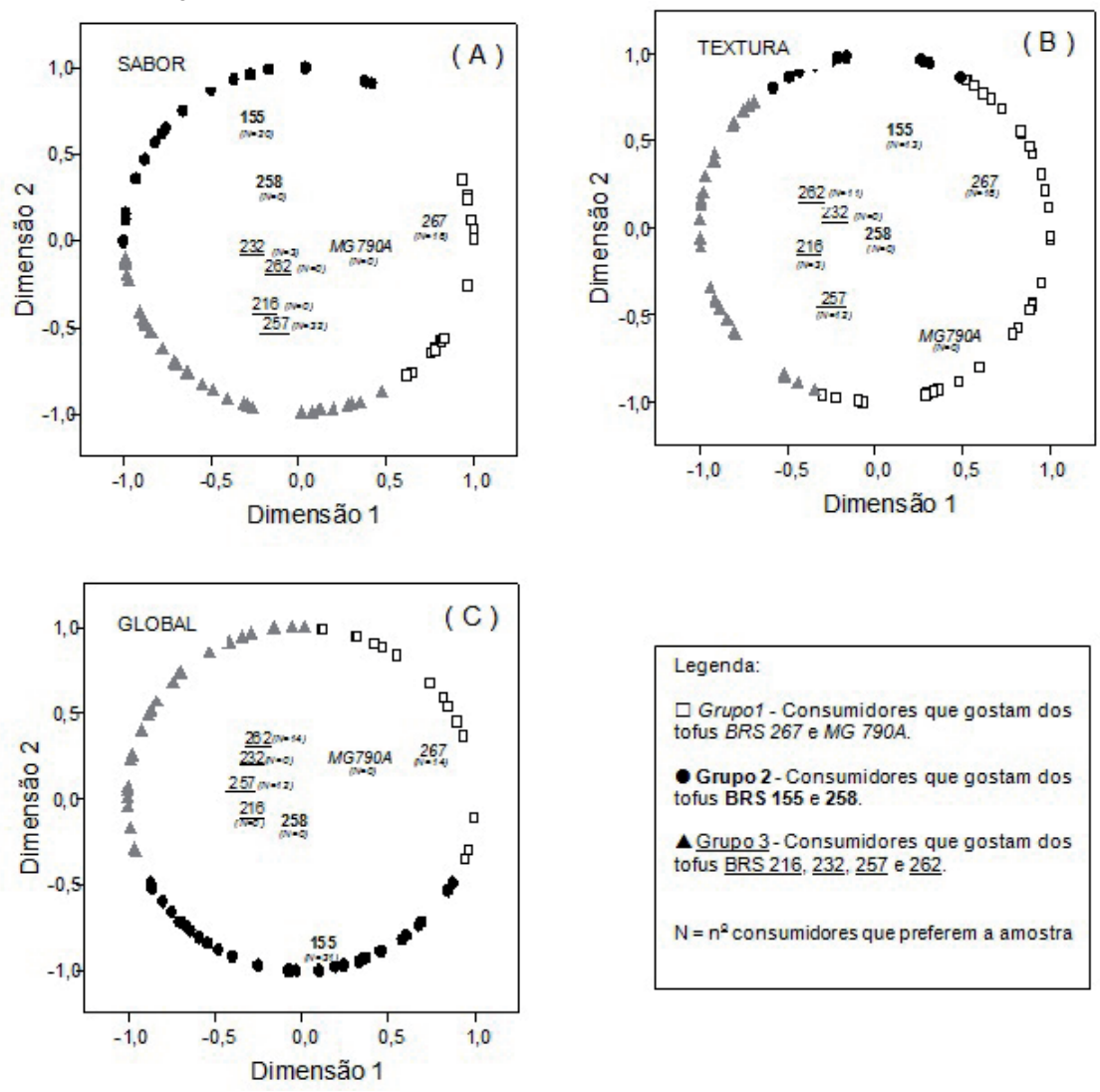
No entanto, a insuficiência ou ausência de sabor característico, considerada positiva por quase metade da equipe, pode ter motivado uma outra parte dos consumidores a gostarem menos do tofu BRS 257, pois associam o sabor característico do produto ao beany flavor. De fato, o termo beany ou "feijão cru" pode ser associado a uma variedade de atributos relacionados entre si e representa uma dificuldade na descrição de produtos de soja, necessitando ser corretamente definido quando empregado na análise sensorial (TORRES-PENARANDA; REITMEIER, 2001). Carrão-Panizzi et al. (1999) verificaram, em extrato e grãos cozidos de soja, que a presença de beanyflavor provocava um mascaramento ou reduzia a percepção de sabor amargo ou adstringência pelos provadores.

Quanto à preferência da textura (Figura 1B), os consumidores do Grupo $1(\mathrm{~N}=16)$ encontramse em oposição aos demais. Os dados de dureza instrumental (Tabela 5) indicaram que os tofus BRS 267 e BRSMG 790A (próximos ao Grupo 1) estão entre as amostras mais macias, enquanto na parte oposta do gráfico situaram-se as amostras mais firmes. A exceção foi o tofu BRS 262, que é macio, mas pode ter sido agrupado junto às amostras mais firmes por outras características de textura que não a dureza, como por exemplo, a suculência ou a granulosidade do produto.

$\mathrm{O}$ atributo aceitação global visou avaliar, em conjunto, todas as percepções sensoriais dos julgadores. Isto pode incluir, além dos atributos avaliados, aroma, cor e outras características que cada julgador considera importante no produto. Embora a análise das médias não tenha indicado diferenças de aceitação global entre as amostras, no Mapa de Preferência Interno (Figura 1C), visualiza-se uma clara divisão no comportamento dos consumidores.

$\mathrm{Na}$ Tabela 7 estão as notas de aceitação global para cada grupo de consumidores. No Grupo 1 estão indivíduos que gostaram dos tofus BRS 267 e BRSMG 790A (médias acima de 6) e rejeitaram os tofus BRS 155 e o 258 (médias abaixo de 5), os quais foram os preferidos pelos consumidores do Grupo 2. O Grupo 3 é constituído por consumidores que rejeitaram os tofus BRS 267 e 155 (média abaixo de 5), os preferidos pelos Grupos 1 e 2, e aceitaram os demais, preferindo o tofu BRS 257.

Tabela 7. Aceitação global de tofus de diferentes cultivares para os grupos de consumidores do Mapa de Preferência Interno* (Figura 1).

\begin{tabular}{llll}
\hline Provador & $\begin{array}{c}\text { Grupo 1 } \\
\text { Tofu }\end{array}$ & $\begin{array}{c}\text { Grupo 2 } \\
(\mathbf{1 5 )}\end{array}$ & $\begin{array}{c}\text { Grupo 3 } \\
(\mathbf{3 1 )}\end{array}$ \\
\hline BRS 155 & $4,5^{\mathrm{c}}$ & $7,3^{\mathrm{a}}$ & $4,5^{\mathrm{c}}$ \\
BRS 216 & $5,3^{\mathrm{abc}}$ & $5,9^{\mathrm{b}}$ & $6,3^{\mathrm{ab}}$ \\
BRS 232 & $5,1^{\mathrm{bc}}$ & $5,5^{\mathrm{b}}$ & $6,4^{\mathrm{a}}$ \\
BRS 257 & $5,3^{\mathrm{abc}}$ & $6,2^{\mathrm{b}}$ & $6,6 \mathrm{a}$ \\
BRS 258 & $4,6^{\mathrm{c}}$ & $6,2^{\mathrm{b}}$ & $5,5^{\mathrm{abc}}$ \\
BRS 262 & $5,1^{\mathrm{bc}}$ & $5,4^{\mathrm{b}}$ & $6,4^{\mathrm{a}}$ \\
BRS 267 & $6,8^{\mathrm{a}}$ & $5,4^{\mathrm{b}}$ & $4,6^{\mathrm{c}}$ \\
BRSMG 790A & $6,3^{\mathrm{ab}}$ & $5,8^{\mathrm{b}}$ & $5,2^{\mathrm{bc}}$ \\
\hline
\end{tabular}

Letras diferentes na mesma coluna indicam diferença significativa (Tukey, $\mathrm{p} \leq 0,05$ )

Valores entre parênteses correspondem ao número de indivíduos em cada grupo

(*) Escala: 1=desgostou extremamente, $5=$ nem gostou, nem desgostou, $9=$ gostou extremamente.

Foi observada correlação significativa $(\mathrm{r}=0,87$, $\mathrm{p} \leq 0,5$ ) entre os resultados de aceitação de sabor e global, ou seja, o sabor exerceu forte influência na aceitação geral do produto. A relação entre o sabor e a aceitação global dos tofus pode ser visualizada também pela distribuição semelhante dos provadores nos Mapas em relação à preferência quanto ao sabor (Figura 1A) e global (Figura 1C).

Foi obtida correlação significativa entre os valores de dureza instrumental e hedônicos de textura $(\mathrm{r}=0,80, \mathrm{p} \leq 0,05)$, indicando que um tofu com maior dureza deveria ter a textura mais apreciada pelos consumidores. Observou-se, no entanto, no Mapa de textura (Figura 1B), uma distribuição diferenciada do global (Figura $1 \mathrm{C}$ ). O tofu BRS 216, caracterizado pela dureza mais alta (Tabela 
6), apresentou boa aceitação para sabor e textura, mas não foi preferido no Mapa global por nenhum consumidor $(\mathrm{N}=0)$. Estes resultados indicam que a dureza do tofu não é o principal atributo considerado na avaliação global.

Foi verificado que o tofu BRS 258 também não foi preferido por nenhum dos consumidores $(\mathrm{N}=0)$ e ocupou sempre posições próximas ao centro dos mapas (Figuras 1A, 1B e 1C). Provavelmente, esta cultivar resulta em um produto com características sensoriais menos marcantes, não obtendo assim a preferência de nenhum grupo. Trabalhando com cinco cultivares brasileiras de soja, Da Silva (2009) avaliou diversas características sensoriais de grãos cozidos, por meio de teste de aceitação de atributos e de Análise Descritiva Quantitativa. A autora observou que a cultivar BRS 258 podia ser considerada como uma cultivar com atributos sensoriais de intensidades intermediárias. Tais resultados sugerem que os atributos do grão são realmente transferidos ao tofu.

Alguns dos conceitos encontrados na literatura sobre as características das cultivares adequadas para tofu não foram confirmados neste trabalho. Por exemplo, que os grãos maiores apresentam menor conteúdo de casca e que, portanto, resultam em maior rendimento de tofu; os resultados mostraram que as cultivares com grãos maiores tinham também o maior conteúdo de cascas e o rendimento dos tofus não foi influenciado por este fato. Também foi verificado que, apesar de grãos com teor mais elevado de proteínas produzirem tofu igualmente mais protéico, isto não afetou o rendimento. Por outro lado, era desconhecida a relação entre a dureza do tofu e sua aceitação pelo consumidor brasileiro; os resultados obtidos mostraram a preferência de textura pelo produto mais firme, embora todos os tofus tenham sido semelhantes em termos de aceitação global.

Os resultados discutidos a partir do Mapa de Preferência Interno podem ser aplicados, de forma prática, por uma empresa fabricante de tofu, quando da definição do tipo de produto a ser colocado no mercado. A empresa pode fabricar um único produto, como o tofu BRS 216, capaz de atender aos requisitos de um público mais numeroso. Por outro lado, a empresa pode optar por comercializar vários produtos diferentes. Por exemplo, produzir um tofu com sabor mais suave, como o BRS 257, e outro (s) com características opostas a este, como os tofus BRS 155 ou 267, atendendo assim a públicos consumidores distintos.

\section{Conclusão}

As cultivares de soja estudadas são distintas em relação ao tamanho, conteúdo de cascas e capacidade de absorção de água e resultam em tofus com diferentes texturas, mas semelhantes quanto ao rendimento. Os tofus das diferentes cultivares apresentam diferenças em termos de sabor e textura, mas não são diferenciados pelos consumidores na aceitação global. Isto indica que todas as cultivares de soja poderiam ser usadas para produção de tofu, com mesmo rendimento e aceitação global. Os consumidores são distribuídos em três grupos distintos de acordo com o Mapa de Preferência interno. Essa segmentação de consumidores em relação aos tofus pode nortear a escolha de uma ou mais cultivares de soja por uma empresa fabricante deste produto, de modo a atender um público consumidor específico.

\section{Referências}

AOAC. Official Methods of Analysis of AOAC International. $16^{\text {th }}$ ed. Arlington: AOAC International, 1995.

BHARDWAJ, H. L.; BHAGSARI, A. S.; JOSHI, J. M.; RANGAPPA, M.; SAPRA, V. T.; RAO, M. S. Yield and quality of soymilk and tofu made from soybean genotypes grown at four locations. Crop Science, Madison, v. 39, n. 2, p. 401-405, 1999.

BENASSI, V. T.; YAMASHITA, F.; PRUDENCIO, S. H. A statistical approach to define some tofu processing conditions. Ciência e Tecnologia de Alimentos, Campinas, v. 31, n. 4, 2011. No prelo. 
CAI, T. D.; CHANG, K. C.; SHIH, M. C.; HOU, H. J.; JI, M. Comparison of bench and production scale methods for making soymilk and tofu from 13 soybean varieties. Food Research International, Barking, v. 30, n. 9, p. 659668, 1997.

CAI, T. D.; CHANG, K. C. Characteristics of productionscale tofu as affected by soymilk coagulation method: propeller blade size, mixing time and coagulant concentration. Food Research International, Barking, v. 31, n. 4, p. 289-295, 1998.

CALEGUER, V. F.; TOFFOLI, C.; BENASSI, M. T. Avaliação da aceitação de preparados sólidos comerciais para refresco sabor laranja e correlação com parâmetros físico-químicos. Semina: Ciências Agrárias, Londrina, v. 27, n. 4, p. 587-598, 2006.

CARRÃO-PANIZZI， M. C.; BELÉIA， A. D. P.; FERREIRA, S. H. P.; OLIVEIRA, M. C. N.; KITAMURA, $\mathrm{K}$. Effects of isoflavones on beany flavor and astringency of soymilk and cooked whole soybeans grains. Pesquisa Agropecuária Brasileira, Brasília, v. 34, n. 6, p. 1045 1052, 1999.

CARRÃO PANIZZI, M. C.; PÍPOLO, A. E.; MANDARINO, J. M. G.; ARANTES, N. E.; GARCIA, A.; BENASSI, V. de T.; ARIAS, C. A.; KASTER, OLIVEIRA, M. F. de; OLIVEIRA, M. A.; TOLEDO, J. F. F. de; MOREIRA, J. U. V.; CARNEIRO, G. E. de S. Breeding specialty soybean cultivars for processing and value-added utilization at Embrapa in Brazil. In: WORLD SOYBEAN RESEARCH CONFERENCE, 8., 2009, Beijing. Developing a global soy blue print for a safe secure and sustainable supply: proceedings. Beijing. Proceedings... Beijing: Chinese Academy of Agricultural Sciences: Institute of Crop Science, 2009a. 1 CD-ROM.

CARRÃO-PANIZZI, M. C.; PÍPOLO, A. E.; MANDARINO, J. M. G.; OLIVEIRA, M. A.; GARCIA, A.; BENASSI, V. de T.; ARIAS, C. A. A.; KASTER, M.; OLIVEIRA, M. F.; TOLEDO, J. F. F.; CARNEIRO, G. E. de S.; MOREIRA, J. U. V.; ARANTES, N. E.; DIAS, W. P.; ALMEIDA, A. M. R.; DOMIT, L. A.; MIRANDA, L. C.; BROGIN, R. L. Cultivar de soja BRS 267 para consumo na alimentação humana como grãos maduros ou verdes como hortaliça. In: CONGRESSO BRASILEIRO DE SOJA MERCOSOJA, 5., 2009, Goiânia. Resumos... Londrina: Embrapa Soja, 2009b. p. 235.

CUI, Z.; JAMES, A. T.; MYIAZAKI, S.; WILSON, R. F.; CARTER JUNIOR, T. E. Breeding specialty soybeans for traditional and new soyfoods. In: LIU, K. (Ed.). Soybeans as functional foods and ingredients. Champaign: AOCS Press, 2004. p. 290-295.

CULTIVARES de soja 2004/2005 Região Centro-Sul. Londrina: Embrapa Soja: Fundação Meridional, 2005. 80 p. (Embrapa Soja. Documentos, 249).

CULTIVARES de soja 2007/2008 Região Centro-Sul. Londrina: Embrapa Soja: Fundação Meridional, 2008. 80 p. (Embrapa Soja. Documentos, 299).

DA SILVA, J. B. Características de cultivares de soja convencionais e para consumo humano: análises físicas, químicas e sensoriais (sentidos humanos e sensores eletrônicos). 2009. Tese (Doutorado em Ciência de Alimentos) - Centro de Ciências Agrárias. Universidade Estadual de Londrina, Londrina.

EVANS, D. E.; TSUKAMOTO, C.; NIELSEN, N. C. A small scale method for the production of soymilk and silken tofu. Crop Science, Madison, v. 37, n. 5, p. $1463-$ 1471, 1997.

HOU, H. J.; CHANG, K. C.; SHIH, M. C. Yield and textural properties of soft tofu as affected by coagulation method. Journal of Food Science, Chicago, v. 62, n. 4, p. 824-827, 1997.

LIM, B. T.; DE MAN, J. M.; DE MAN, L.; BUZZEL R. I. Yield and quality of tofu as affected by soybean and soymilk characteristics: calcium sulfate coagulant. Journal of Food Science, Chicago, v. 55, n. 4, p. 10881092, 1990.

LIU, K. Soybeans: chemistry, technology and utilization. Gaithersburg: Aspen Publishers, 1999.

MEILGAARD, M. C.; CIVILlE, G. V.; CARR, T. Sensory evaluation techniques. $4^{\text {th }}$ ed. New York: CRC Press, 2007.

MIN, S.; YU, Y.; ST. MARTIN, S. Effect of soybean varieties and growing locations on the physical and chemical properties of soymilk and tofu. Journal of Food Science, Chicago, v. 70, n. 1, p. 8-12, 2005.

MULLIN, W. J.; FREGEAU-REID, J. A.; BUTLER, M.; POYSA, V.; WOODROW, L.; JESSOP, D. B.; RAYMOND, D. An interlaboratory test of a procedure to assess soybean quality for soymilk and tofu production. Food Research International, Barking, v. 34, n. 8, p. 669677, 2001.

MURPHY, P. A.; CHEN, H. P.; HAUCK, C. C.; WILSON, L. A. Soybean protein composition and tofu quality. Food Technology, Chicago, v. 51, n. 3, p. 86-88, 110, 1997.

OLIVEIRA, A. P. V.; FRASSON, K.; ALMEIDA, T. C. A.; BENASSI, M. T. Aceitação de sobremesas lácteas dietéticas e formuladas com açúcar: teste afetivo e mapa de preferência interno. Ciência e Tecnologia de Alimentos, Campinas, v. 24, n. 4, p. 627-633, 2004.

OP\&P PRODUCT RESEARCH. Senstools versão 2.3. Utrecht: OP\&P Product Research, 1995-1998. Conjunto de programas. 1 CD-ROM. 
POYSA, V.; WOODROW, L. Stability of soybean seed composition and its effect on soymilk and tofu yield and quality. Food Research International, Barking, v. 35, n. 4, p. 337-345, 2002.

POYSA, V.; WOODROW, L.; YU, K. Effect of soy protein subunit composition on tofu quality. Food Research International, Barking, v. 39, n. 3, p. 309-317, 2006.

ROBINSON, D. S.; WU, Z.; DOMONEY, C.; CASEY, R. Lipoxigenases and the quality of foods. Food Chemistry, Davis, v. 54, n. 1, p. 33-43, 1995.

ROSSET, M. Distribuição de ácido fitico e minerais durante o processamento de extrato hidrossolúvel de soja e tofu. 2007. Dissertação (Mestrado em Ciência de Alimentos) - Centro de Ciências Agrárias. Universidade Estadual de Londrina, Londrina.

SHEN, C. F.; DE MAN, L.; BUZZEL R. I.; DE MAN, J. $M$. Yield and quality of tofu as affected by soybean and soymilk characteristics: glucono-delta-lactone coagulant. Journal of Food Science, Chicago, v. 56, n. 1, p. 109-112, 1991.

STATSOFT. Statistica for Windows. Computer program manual. Tulsa: Statsoft, Inc., 1995.

TORRES-PENARANDA, A. V.; REITMEIER, C. A. Sensory descriptive analysis of soymilk. Journal Food Science, Chicago, v. 66, n. 2, p. 352 - 356, 2001.

TORRES-PENARANDA, A. V.; REITMEIER, C. A.; WILSON, L. A.; FEHR, W. R.; NARVEL, J. M. Sensory Characteristics of Soymilk and Tofu made from Lipoxygenase-Free and Normal Soybeans. Journal Food Science, Chicago, v. 63, n. 6, p. 1084-1087, 1998.

WANG, H. L.; SWAIN, E. W.; KWOLEK, W. F. Effect of soybean varieties on the yield and quality of tofu. Cereal Chemistry, St. Paul, v. 60, n. 3, p. 245-248, 1983.

YOKOMIZO, G. K.; DUARTE, J. B.; VELLO, N. A. Correlações fenotípicas entre tamanho de grãos e outros caracteres em topocruzamentos de soja tipo alimento com tipo grão. Pesquisa Agropecuária Brasileira, Brasília, v. 35, n. 11, p. 2235-2241, 2000. 